A. Olevskii, School of Mathematical Sciences, Faculty of Science, University of Tel Aviv, Israel. e-mail: olevskii@@math.tau.ac.il

C. E. Weil, Department of Mathematics, Michigan State University,

East Lansing, Mi 48824-1027, USA. e-mail:weil@@math.msu.edu

\title{
A CONTINUOUS FUNCTION NOT TWICE PEANO DIFFERENTIABLE ON ANY PERFECT SET
}

\begin{abstract}
An example of a continuous function is given that is differentiable except on a countable set, but is not twice Peano differentiable on any nonempty, perfect set.
\end{abstract}

\section{Introduction.}

In 1984 M. Laczkovich [2] proved that for every continuous, real valued function of one real variable there is a nonempty, perfect set with respect to which the function is infinitely differentiable. In a way this result indicates how ineffective the classical notion of higher order differentiation is when it is applied to non connected subsets. In particular, knowledge of the classical higher order derivatives of a function computed relative to a (non connected) set gives no information about the behavior of the function as was shown by Maŕík in [3]. There he gave an example of a perfect set, $H$, with $0 \in H$ and a function, $f$, defined on $H$ that behaves about like $x^{2}$ near 0 , but with $f^{(k)}(0)=0$ for all $k \in \mathbb{N}$. However, a function behaving like $x^{2}$ near 0 ought to have $f^{\prime}(0)=0$ and $f^{(2)}(0)=2$ since these are the first and second derivatives of $x^{2}$ at 0 . The notion of higher order differentiation that gives these correct derivatives to Mař́k's example is the Peano notion.

Key Words: Peano differentiation

Mathematical Reviews subject classification: 26A24

Received by the editors September 1, 1995 
Definition 1.1 Let $f: H \rightarrow \mathbb{R}$ and let $x \in H$. Then $f$ is $k$ times Peano differentiable at $x$ relative to $H$ means there are $k$ numbers denoted by $f_{1}(x), f_{2}(x)$, $\ldots, f_{k}(x)$ and a function $\varepsilon_{x}: H \rightarrow \mathbb{R}$ such that $\lim _{\substack{y \rightarrow x \\ y \in H}} \varepsilon_{x}(y)=\varepsilon_{x}(x)=0$ and for $y \in H$

$$
f(y)=f(x)+\sum_{i=1}^{k} \frac{f_{i}(x)}{i !}(y-x)^{i}+(y-x)^{k} \varepsilon_{x}(y) .
$$

The reader unfamiliar with the concept of Peano differentiation can learn more about it by consulting either of the two survey articles, [1] or [5]. For the purposes of this article the reader should be aware that the existence of $f^{(k)}(x)$ computed relative to a non connected set $H$ doesn't imply the existence of $f_{k}(x)$ computed relative to $H$. Moreover even when both $f^{(k)}(x)$ and $f_{k}(x)$ exist, they need not be equal. In light of these observations it is reasonable to ask if the assertion resulting from the Laczkovich result mentioned at the outset by replacing the classical notion of higher order differentiation with the Peano notion is true. We show by example that it is not.

\section{The Example}

In this section we construct a function, $f$, defined on $[0,1]$, a countable set, $E \subset[0,1]$, and a number $c>0$ such that $f$ is differentiable on $[0,1] \backslash E$ and if $x, y \in[0,1] \backslash E$, then

$$
\begin{aligned}
& \left|f(y)-f(x)-(y-x) f^{\prime}(x)\right| \geq c|y-x|^{\frac{3}{2}} \\
& \text { or } \\
& \left|f(x)-f(y)-(x-y) f^{\prime}(y)\right| \geq c|y-x|^{\frac{3}{2}} .
\end{aligned}
$$

The example is a modification of one presented by the first author in [4].

Before constructing the function, it will be shown that for such a function there is no nonempty perfect set relative to which it is twice Peano differentiable. For suppose there is a nonempty perfect set, $H$, such that for each $x \in H$

$$
\lim _{\substack{y \rightarrow x \\ y \in H}} \frac{f(y)-f(x)-(y-x) f^{\prime}(x)-\frac{(y-x)^{2}}{2} f_{2}(x)}{(y-x)^{2}}=0 .
$$

Then clearly for each $x \in H$

$$
\lim _{\substack{y \rightarrow x \\ y \in H}} \frac{f(y)-f(x)-f^{\prime}(x)(y-x)}{(y-x)^{\frac{3}{2}}}=0 .
$$


Now by the Baire Category Theorem, there is a $\delta>0$ and an interval $(a, b)$ with $b-a<\delta$ such that $H \cap(a, b) \neq \emptyset$ and

$$
\left\{x \in H \cap(a, b): \frac{\left|f(y)-f(x)-f^{\prime}(x)(y-x)\right|}{|y-x|^{\frac{3}{2}}}<c \text { for all } y \in H \text { with }|y-x|<\delta\right\}
$$

is uncountable and dense in $H \cap(a, b)$. Choosing $x$ and $y$ in the above set that don't belong to $E$ contradicts (2).

Let

$$
r(t)= \begin{cases}1 & \text { if } t \in(n, n+1) \text { with } n \in \mathbb{N} \text { even } \\ -1 & \text { if } t \in(n, n+1) \text { with } n \in \mathbb{N} \text { odd } \\ 0 & \text { if } t \in \mathbb{N} \cup\{0\} .\end{cases}
$$

For $x \in[0,1]$ let

$$
f(x)=\int_{0}^{x} \sum_{n=1}^{\infty} 8^{-n} r\left(2^{6 n} t\right) d t
$$

and set $E=\left\{\frac{j}{2^{6 n}}: n \in \mathbb{N}, j=0,1, \ldots 2^{6 n}\right\}$. We will show that this function has the desired properties. First it is clear that $\sum_{n=1}^{\infty} 8^{-n} r\left(2^{6 n} x\right)$ is continuous on $[0,1] \backslash E$ and hence for $x \in[0,1] \backslash E$ we have $f^{\prime}(x)=\sum_{n=1}^{\infty} 8^{-n} r\left(2^{6 n} x\right)$. To prove the assertions about $f$, it is convenient to refer to the intervals $\left[\frac{j-1}{2^{6 n}}, \frac{j}{2^{6 n}}\right)$ for $j=1,2, \ldots, 2^{6 n}$ as the intervals of rank $n$ for $n \in \mathbb{N}$. Let $x, y \in[0,1] \backslash E$ with $y \neq x$. Then there is a unique $\ell \in \mathbb{N}$ such that for $n \leq \ell-1, x$ and $y$ lie in the same interval of rank $n$ but in different intervals of rank $\ell$. Then

$$
\begin{aligned}
f(y)-f(x)-(y-x) f^{\prime}(x)= & \int_{x}^{y} 8^{-\ell}\left(r\left(2^{6 \ell} t\right)-r\left(2^{6 \ell} x\right)\right) d t \\
& +\int_{x}^{y} \sum_{n=\ell+1}^{\infty} 8^{-n}\left(r\left(2^{6 n} t\right)-r\left(2^{6 n} x\right)\right) d t \\
& =T_{1}+T_{2} .
\end{aligned}
$$

The second term is the easier to estimate.

$$
\left|T_{2}\right| \leq 2|y-x| \sum_{n=\ell+1}^{\infty} 8^{-n}=\frac{2}{7} 8^{-\ell}|y-x| .
$$

To obtain the desired estimate on the first term, two cases are considered. First assume that $x$ and $y$ don't lie in neighboring intervals of rank $\ell$. Then 
it isn't hard to see that $\left|T_{1}\right| \geq 8^{-\ell} \frac{2}{3}|y-x|$. Thus

$$
\left|f(y)-f(x)-(y-x) f^{\prime}(x)\right| \geq\left|T_{1}\right|-\left|T_{2}\right| \geq \frac{8}{21} 8^{-\ell}|y-x| .
$$

Since $|y-x|<2^{-6(\ell-1)}=2^{6}\left(8^{-\ell}\right)^{2}$,

$$
\left|f(y)-f(x)-(y-x) f^{\prime}(x)\right| \geq \frac{1}{21}|y-x|^{\frac{3}{2}} .
$$

Second and finally suppose that $x$ and $y$ lie in neighboring intervals of

rank $\ell$. Then $|y-x|<2^{-6 \ell+1}$. Let $a=\frac{j}{2^{6 \ell}}$ lie between $x$ and $y$. Suppose $|y-a| \geq|x-a|$. (In the opposite case, reverse the roles of $x$ and $y$.) Then $\left|T_{1}\right|=8^{-\ell} 2|y-a| \geq 8^{-\ell}|y-x|$. Since $8^{-\ell}=\left(2^{-6 \ell}\right)^{\frac{1}{2}}>2^{-\frac{1}{2}}|y-x|^{\frac{1}{2}}$,

$\left|f(y)-f(x)-(y-x) f^{\prime}(x)\right| \geq 8^{-\ell}|y-x|-\frac{2}{7} 8^{-\ell}|y-x| \geq \frac{5}{7} 8^{-\ell}|y-x|>\frac{5}{7 \sqrt{2}}|y-x|^{\frac{3}{2}}$.

Consequently the assertion is true with $c=\frac{1}{21}$.

\section{References}

[1] M. J. Evans and C. E. Weil, Peano Derivatives: A Survey, Real Analysis Exch., 7 (1981-82), 5-24.

[2] M. Laczkovich, Differentiable restrictions of continuous functions, Acta Math. Hungar., 44 (1984), 363-372.

[3] J. Mařík, Derivatives and closed sets, Acta Math. Acad. Sci. Hungar., 43 (1984), 25-29.

[4] A. Olevskii, Ulam-Zahorski problem on free interpolation by smooth functions, Trans. Amer. Math. Soc., 342 (1994), 713-727.

[5] C. E. Weil, The Peano Notion of Higher Order Differentiation, Math Japonica, 42, 587-600. 